\title{
MORFOLOGÍA DE LA SEMILLA EN NEOBUXBAUMIA (CACTACEAE)
}

\author{
Gabriel Arroyo-Cosultchi' ${ }^{1}$, Teresa Terrazas²,4, Salvador Arias ${ }^{3}$ y Lauro López-Mata ${ }^{1}$ \\ 'Programa en Botánica, Colegio de Postgraduados, Montecillo, Edo. de México, C.P. 56230, México. \\ ${ }^{2}$ Departamento de Botánica, Instituto de Biología, Universidad Nacional Autónoma de México, \\ Apdo. Postal 70-233, México 04510, D.F., México. \\ 3Jardín Botánico, Instituto de Biología, Universidad Nacional Autónoma de México, \\ Apdo. Postal 70-614, México 04510, D.F., México. \\ ${ }^{4}$ Autor para la correspondencia. Correo-e: tterrazas@ibiologia.unam.mx
}

\begin{abstract}
Resumen: La morfología de semillas de nueve especies de Neobuxbaumia y Carnegiea gigantea fue examinada mediante microscopía electrónica de barrido con la finalidad de contrastar sus similitudes y diferencias. Se evaluaron 14 caracteres cualitativos y cuantitativos para identificar grupos de especies, usando un análisis de similitud. Se distinguieron dos grupos con base en la forma de la semilla y la posición relativa a la región hilo-micropilar. Las especies de Neobuxbaumia presentaron semillas largas y muy largas, asimétricas, lustrosas, lisas, con quilla en la periferia; la región hilo-micropilar presenta el borde ligeramente expandido alrededor del hilo, oblicuo, superficial y el complejo de forma ovalado. Neobuxbaumia multiareolata tiene semillas con intersticios y en $N$. laui la escultura de la pared periclinal presenta convexidad de domos altos. Carnegiea gigantea se diferencia de las especies de Neobuxbaumia por sus semillas ovoides, el borde de su testa ampliamente expandido alrededor del hilo, la posición relativa a la región hilo micropilar superficial y el complejo en forma de cerradura. Varios de los rasgos señalados se comparten con otros miembros de Pachycereinae y no se identifican caracteres de la semilla únicos para las especies de Neobuxbaumia que puedan ser considerados como posibles sinapomorfias.
\end{abstract}

Palabras clave: Cactaceae, microscopio electrónico de barrido, Norteamérica, Pachycereinae, quilla, similitud, testa.

\begin{abstract}
Seed morphology of nine species of Neobuxbaumia and Carnegiea gigantea was examined by scanning electronic microscopy to distinguish their similarities and differences. Fourteen quantitative and qualitative features were evaluated to identify groups of species using a phenetic analysis. Two groups were recognized based on seed shape and rim position relative to the hilum-micropylar region. Neobuxbaumia species are characterized by the following features: large to extremely large, glossy, smooth seeds, with periphery keeled, border slightly expanded around hilum, hilum-micropylar region oblique, superficial, and hilum-micropyle complex oval. Neobuxbaumia multiareolata has obvious interstices and $N$. laui possesses convexities with high domes. Carnegiea gigantea differs from Neobuxbaumia species in that its seeds are ovoid, with border highly expanded around hilum, hilum-micropylar region superficial, and hilum-micropyle complex key-shaped. Most seed characters described are shared with member of Pachycereinae, and thus no single seed character can be recognized as a synapomorphy for Neobuxbaumia. Key words: Cactaceae, keel, North America, Pachycereinae, scanning electronic microscopy, similarity, testa.
\end{abstract}

$\mathbf{E}_{\mathrm{t}}^{1}$ género Neobuxbaumia Backeberg es un miembro de la familia Cactaceae que se distribuye en ambientes secos y semi-húmedos de la Depresión del Balsas, el Valle de Tehuacán-Cuicatlán, la vertiente del Pacífico y la vertiente del Golfo de México (Bravo-Hollis, 1978). Neobuxbaumia comprende plantas columnares o con ramas escasas $(N$. tetetzo) o numerosas ( $N$. scoparia), tallos cilíndricos con pocas (8-10 en $N$. euphorbioides) a numerosas (29-31 en $N$. laui) costillas; flores tubulares-campanuladas $(N$. macrocephala, N. multiareolata, N. polylopha, N. scopa - ria) o tubulares-infundibuliformes (N. euphorbioides, N. mezcalaensis, $N$. squamulosa, $N$. tetetzo), nocturnas; frutos ovoides con espinas, dehiscencia vertical en forma de estrella y pulpa blanca y semi-seca (Bravo-Hollis, 1978; Hunt, 2006).

El número de especies que conforman Neobuxbaumia varía de ocho a nueve (Guzmán et al., 2003; Hunt, 2006). Hunt y Taylor (1990) consideraron a Neobuxbaumia como un sinónimo de Carnegiea y Heath (1992) propuso la nueva combinación transfiriendo a todas las especies de 


\section{Gabriel ARroyo-Cosultchi et aL.}

Neobuxbaumia a Carnegiea. Recientemente, la evidencia molecular reveló que Carnegiea es el género hermano de Pachycereus y que no está cercanamente relacionado con Neobuxbaumia (Arias et al., 2003, 2005). Sin embargo, las relaciones entre las especies de Neobuxbaumia están pobremente entendidas. Por ello, aquí se estudia la morfología de la semilla con la finalidad de identificar caracteres que permitan distinguirlas y reconocer si existen diferencias con otros miembros de Pachycereinae, especialmente Carnegiea.

Barthlott y Hunt (2000) publicaron la descripción de las semillas de dos especies de este género, $N$. euphorbioides y N. tetetzo, mientras que Scheinvar y Sánchez-Mejorada (1990) y Lau (1994) incluyeron microfografías de las semillas de $N$. squamulosa y $N$. sanchezmejoradae (= N. laui) como parte de la descripción de los nuevos taxones, respectivamente.

El propósito del presente estudio fue describir la superficie de la semilla de las nueve especies de Neobuxbaumia mediante el análisis de imágenes generadas por el microscopio electrónico de barrido, así como compararla con la de las semillas de Carnegiea gigantea y otros miembros de Pachycereinae. Además, la similitud de la morfología de la semilla se evaluó a través de un análisis fenético, en el que también fueron incluidos Carnegiea gigantea y Pachycereus weberi (J.M.Coult.) Backeb., con el objetivo de identificar si existen caracteres de la semilla que separen las especies de Neobuxbaumia de las de Carnegiea gigan tea y de Pachycereus weberi.

\section{Materiales y métodos}

Se recolectaron semillas maduras de las nueve especies de Neobuxbaumia y de Carnegiea gigantea de poblaciones silvestres o se removieron de ejemplares de herbario (cuadro 1); la información de Pachycereus weberi se tomó de Arias y Terrazas (2004). Las semillas fueron examinadas con el microscopio estereoscópico y el microscopio electrónico de barrido (MEB). Las observaciones en el microscopio estereoscópico permitieron seleccionar por fruto las semillas sanas y turgentes para describir su testa y su morfometría. Para su observación en el MEB (Hitachi-S$2460 \mathrm{~N}$ ), dos o tres semillas por especie se lavaron con agua destilada utilizando ultrasonido, se fijaron a un porta-muestra de aluminio con pegamento a base de carbono y se cubrieron con oro (Hitachi-S-2460N). Las fotografías de la morfología y micromorfología se produjeron en formato de $35 \mathrm{~mm}$ con el MEB. Como las células de la testa pueden variar dependiendo de la región de la semilla observada, todos los acercamientos se tomaron en la parte central de la región lateral (Barthlott y Voit, 1979).

Los caracteres evaluados en la testa fueron la apariencia (color, lustre, periferia y borde), el patrón celular (formas de la célula en región lateral), los límites de la pared anticlinal (relieve, curvatura de la pared e intersticios), la escultura de la pared periclinal (relieve, convexidad y microrelieve) y la región hilo micropilar (RHM: orientación, posición relativa al borde, configuración y forma del complejo). Los caracteres se describen de acuerdo con

Cuadro 1. Especies examinadas en este estudio. *Especimenes usados en el MEB figura 2-5. Los acrónimos de los herbarios se indican entre paréntesis, seguidos del colector y número. Semillas obtenidas de ejemplar de herbario.

\begin{tabular}{|c|c|c|}
\hline Especies & País y estado & Ejemplares de herbario \\
\hline Neobuxbaumia euphorbioides (Haw.) Buxb. ex Bravo & $\begin{array}{l}\text { México: San Luis Potosí, } \\
\text { Tamaulipas, Veracruz }\end{array}$ & Flores s.n. (IZTA)* \\
\hline N. laui (P.V.Heath) D.R.Hunt & México: Oaxaca & $\begin{array}{l}\text { Jardín Etnobotánico de Oaxaca } \\
\left(095,1417^{*}\right)\end{array}$ \\
\hline N. macrocephala (F.A.C.Weber ex K.Schum.) E.Y.Dawson & México: Puebla & Flores s.n. (IZTA)* \\
\hline N. mezcalaensis (Bravo) Backeb. & $\begin{array}{l}\text { México: Guerrero, Puebla, } \\
\text { Morelos, Oaxaca }\end{array}$ & $\begin{array}{l}\text { Arroyo-Cosultchi } 16 \text { (MEXU, CHAPA) * } \\
\text { Flores s.n.( ZTA) }\end{array}$ \\
\hline $\begin{array}{l}\text { N. multiareolata (E.Y.Dawson) Bravo, Scheinvar } \\
\text { et Sánchez-Mej. }\end{array}$ & México: Guerrero & $\begin{array}{l}\text { Arroyo-Cosultchi } 8 \text { (MEXU, CHAPA) * } \\
\text { Flores s.n. (IZTA) }\end{array}$ \\
\hline N. polylopha (DC.) Backeb. & $\begin{array}{l}\text { México: Guanajuato, Hidalgo, } \\
\text { Querétaro, San Luis Potosí }\end{array}$ & $\begin{array}{l}\text { Arroyo-Cosultchi } 22 \text { (MEXU, CHAPA)* } \\
\text { Flores s.n. (IZTA)* }\end{array}$ \\
\hline N. scoparia (Poselg.) Backeb. & México: Oaxaca, Veracruz & Bravo-Hollis $71($ MEXU)* Flores s.n. (IZTA) \\
\hline N. squamulosa Scheinvar et Sánchez-Mej. & $\begin{array}{l}\text { México: Colima, Guerrero, } \\
\text { Jalisco, Michoacán, Oaxaca }\end{array}$ & $\begin{array}{l}\text { Arroyo-Cosultchi } 15 \text { (MEXU, CHAPA) } \\
\text { Flores s.n. (IZTA)* }\end{array}$ \\
\hline N. tetetzo (F.A.C.Weber ex K.Schum.) Backeb. & México: Oaxaca, Puebla & S. Arias 831 (MEXU)* \\
\hline Carnegiea gigantea (Engelm.) Britton et Rose & $\begin{array}{l}\text { México: Sonora } \\
\text { Estados Unidos: Arizona }\end{array}$ & Pringle s.n. (MEXU)* \\
\hline
\end{tabular}


Cuadro 2. Caracteres y estados de carácter de semillas.

\begin{tabular}{|c|c|}
\hline Caracter & Descripción \\
\hline 1 & Forma de semilla: ovoide (OV), ovada (OB) \\
\hline 2 & Color: negro $(\mathrm{N})$, café oscuro $(\mathrm{CO})$ \\
\hline 3 & Posición de la quilla: apical (A), ventral (V) \\
\hline 4 & Borde de la testa: ligeramente expandido alrededor del hilo (LE), ampliamente expandido alrededor del hilo (AE) \\
\hline 5 & Forma de la célula: isodiamétrica poligonal (IP), alargada poligonal (AP) \\
\hline 6 & Límites anticlinales: invisibles (I), acanalados (AC), ligeramente levantados (LL) \\
\hline 7 & Escultura de la pared periclinal: plana (P), domo bajo (DB), domo alto (DA) \\
\hline 8 & Posición relativa a la RHM: superficial (S), finamente impresa (FI) \\
\hline 9 & Forma del complejo HM: ovalada (O), tipo “cerradura" (CE). \\
\hline 10 & Largo: $>2.5 \mathrm{~mm}(0),<2.5 \mathrm{~mm}(1)$ \\
\hline 11 & Ancho: $>2.0 \mathrm{~mm}(0),<2.0 \mathrm{~mm}(1)$ \\
\hline 12 & Área: $>4.00 \mathrm{~mm}^{2}(0),<4.00 \mathrm{~mm}^{2}(1)$ \\
\hline 13 & Intersticios: ausentes $(\mathrm{A})$, presentes $(\mathrm{P})$ \\
\hline 14 & Microrelieve: liso (L), estriaciones finas (EF) \\
\hline
\end{tabular}
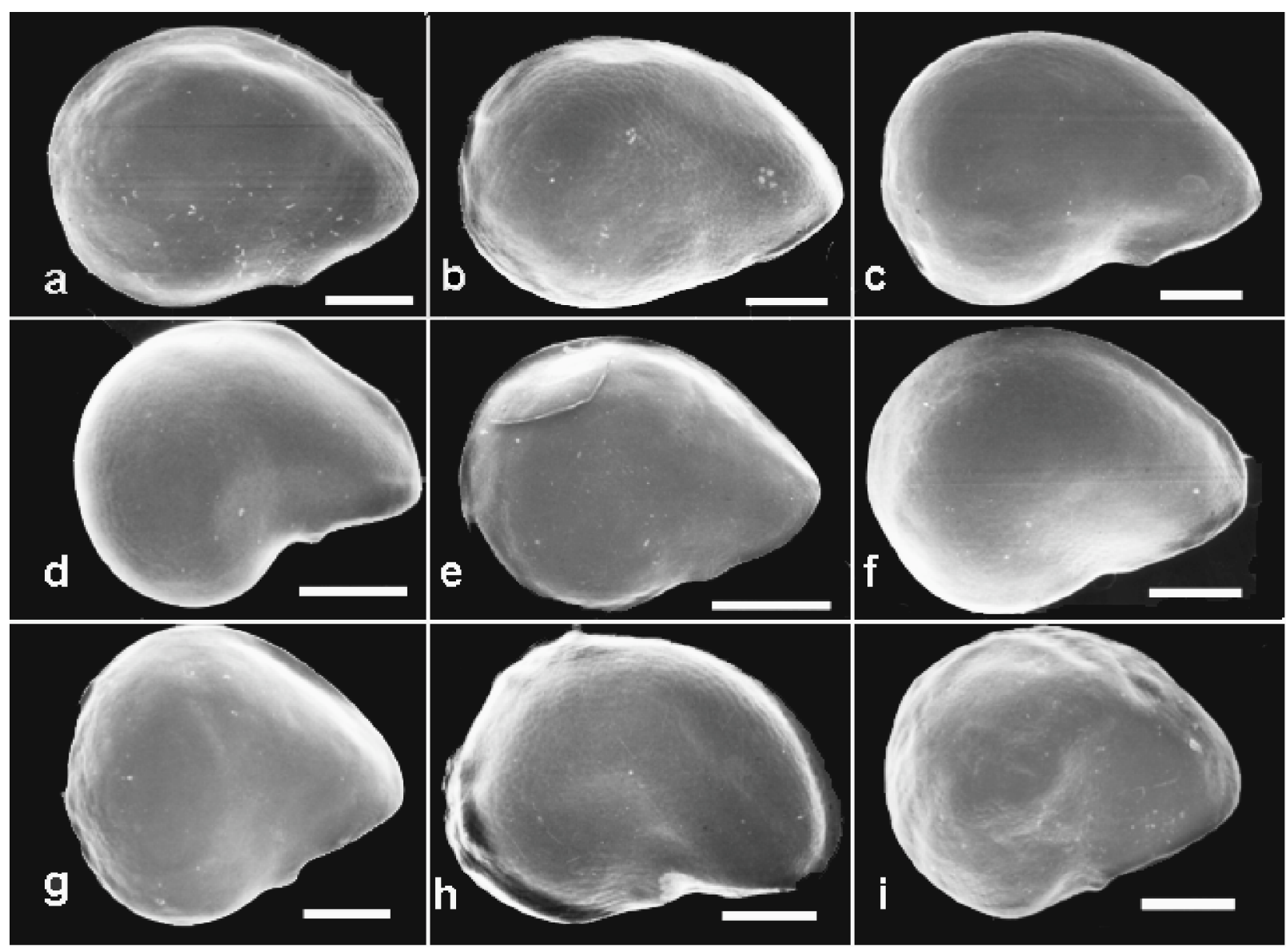

Figura 1. Microfotografías MEB de la semilla madura. a. Neobuxbaumia macrocephala, Flores s.n. (IZTA). b. N. scoparia, Bravo-Hollis 71 (MEXU). c. N. euphorbioides, Flores s.n. (IZTA). d. N. polylopha, Arroyo-Cosultchi 22 (CHAPA). e. N. squamulosa, Flores s.n. (IZTA). f. N. tetetzo, S. Arias 831 (MEXU). g. N. multiareolata, Arroyo-Cosultchi 8 (CHAPA). h. N. mezcalaensis, Flores s.n. (IZTA). i. $N$. laui, Jardín Etnobotánico de Oaxaca 1417. Escalas: a-c $=500$ m, d-i $=1 \mathrm{~mm}$. 

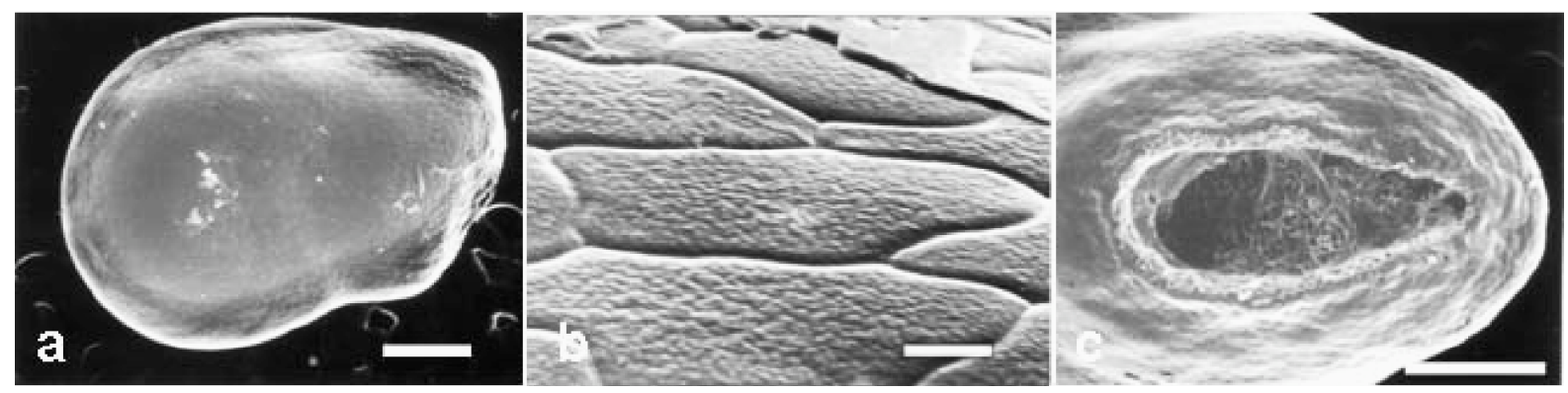

Figura 2. Microfotografías MEB de la semilla de Carnegiea gigantea, Pringle s.n. (MEXU). a. vista general. b. vista de la célula en región lateral. c. región hilo-micropilo. Escala: $\mathrm{a}=500 \mathrm{~m}, \mathrm{~b}=50 \mathrm{~m}, \mathrm{c}=300 \mathrm{~m}$.

Barthlott y Hunt (2000).

El largo, el ancho y el área de la semilla se cuantificaron con un analizador de imágenes (Image-Pro Plus Versión 3.1, Media Cybernetics, 1997) conectado a un microscopio Olympus SZ-ST. La media y el error estándar se calcularon con base en 50 semillas por especie usando el paquete estadístico SAS (SAS Institute, 1989). Además, el valor de la relación largo/ancho de la semilla permitió asignar la forma de la semilla.

La similitud en la morfología de las semillas se evaluó a partir de 14 caracteres (cuadro 2). Doce caracteres fueron binarios y dos carácter multiestado; los primeros fueron codificados como estados excluyentes (es decir, presenciaausencia) y los últimos transformando cada estado en datos presencia-ausencia (Sneath y Sokal, 1973; Crisci y LópezArmengol, 1983). Con ellos se construyó una matriz con 11 taxones y 30 caracteres (apéndice 1). La matriz de similitud se generó con el coeficiente de asociación de Jaccard y el fenograma se produjo con el método de agrupamiento.

\section{Resultados}

Morfología. Morfología general y apariencia de la testa.Neobuxbaumia y Carnegiea tienen semillas asimétricas, de forma ovoide en todas las especies de Neobuxbaumia y ovada en $C$. gigantea (figuras 1,2 ). Los tamaños varían de mediano en $C$. gigantea $\left(\begin{array}{ll}1.90 & 1.40 \mathrm{~mm}\end{array}\right)$ a largo en siete especies de Neobuxbaumia y muy largo en $N$. mezcalaensis (4.11 $2.97 \mathrm{~mm})$ y N. laui $(4.00 \quad 3.00 \mathrm{~mm}$ ) (cuadro 3). El área de la semilla tiene valores de la media desde 2.81 $\mathrm{mm}^{2}$ en $N$. scoparia hasta $8.94 \mathrm{~mm}^{2}$ en $N$. mezcalaensis (cuadro 3). Las semillas son de color café oscuro en N. euphorbioides, $N$. mezcalaensis, $N$. multiareolata y N. tetetzo, y negro en las otras cinco especies de Neobuxbaumia y $C$. gigantea (cuadro 3). Todas las semillas estudiadas presentan una superficie lustrosa (figuras 1,2). Una quilla está presente en la región dorsal y ésta puede terminar en la región apical o hasta la región ventral, como sucede en cinco especies de Neobuxbaumia y C. gigantea (cuadro 3; figuras 1,2 ). Además, las células mantienen su tamaño y forma desde la región lateral hasta la RHM (figuras 1,2) y el borde de la RHM está ligeramente expandido en las especies de Neobuxbaumia y ampliamente expandido en $C$. gigantea (figuras 1,2).

Patrón de las células de la testa.-Las células son de forma isodiamétrica-poligonal en la región lateral, excepto en $N$. euphorbioides, $N$. macrocephala y $C$. gigantea, las cuales tienen las células de forma alargada-poligonal (cuadro 3; figuras 2, 3). El relieve del límite de la pared anticlinal es invisible en varias especies (cuadro 4), ligeramente eleva-

Cuadro 3. Caracteres cualitativos y cuantitativos (media \pm error estándar) de la morfología y de la apariencia de la testa de las semillas de Neobuxbaumia y Carnegiea.

\begin{tabular}{|c|c|c|c|c|c|c|c|}
\hline Especie & $\begin{array}{l}\text { Largo } \\
\qquad(\mathrm{m}\end{array}$ & $\begin{array}{l}\text { ancho } \\
\text { m) }\end{array}$ & $\begin{array}{l}\text { Área } \\
\left(\mathrm{mm}^{2}\right)\end{array}$ & Color & $\begin{array}{l}\text { Posición de } \\
\text { la quilla }\end{array}$ & $\begin{array}{l}\text { Borde de } \\
\text { la testa }\end{array}$ & $\begin{array}{l}\text { Forma de } \\
\text { la célula }\end{array}$ \\
\hline N. euphorbioides & $2.43 \pm 0.03$ & $1.70 \pm 0.04$ & $3.31 \pm 0.06$ & $\mathrm{CO}$ & D-A & LE & LP \\
\hline N. laui & $4.00 \pm 0.01$ & $3.00 \pm 0.01$ & $9.00 \pm 0.02$ & $\mathrm{~N}$ & D-A & LE & LP \\
\hline N. macrocephala & $2.15 \pm 0.01$ & $1.56 \pm 0.00$ & $2.86 \pm 0.02$ & $\mathrm{~N}$ & $D-A-V$ & LE & LP \\
\hline N. mezcalaensis & $3.78 \pm 0.03$ & $2.79 \pm 0.02$ & $8.94 \pm 0.10$ & $\mathrm{CO}$ & D-A-V & LE & IP \\
\hline N. multiareolata & $3.25 \pm 0.02$ & $2.69 \pm 0.02$ & $6.56 \pm 0.08$ & $\mathrm{CO}$ & D-A-V & LE & IP \\
\hline N. polylopha & $2.68 \pm 0.04$ & $1.85 \pm 0.02$ & $4.01 \pm 0.05$ & $N$ & D-A & LE & IP \\
\hline N. scoparia & $2.21 \pm 0.01$ & $1.63 \pm 0.00$ & $2.81 \pm 0.01$ & $\mathrm{~N}$ & D-A-V & LE & IP \\
\hline N. squamulosa & $2.78 \pm 0.01$ & $2.15 \pm 0.01$ & $4.86 \pm 0.02$ & $\mathrm{~N}$ & $D-A-V$ & LE & IP \\
\hline N. tetetzo & $2.95 \pm 0.02$ & $2.43 \pm 0.02$ & $5.17 \pm 0.06$ & $\mathrm{CO}$ & D-A & LE & IP \\
\hline C. gigantea & $1.90 \pm 0.01$ & $1.40 \pm 0.01$ & $2.66 \pm 0.03$ & $\mathrm{~N}$ & D-A-V & $\mathrm{AE}$ & LP \\
\hline
\end{tabular}


Cuadro 4. Patrón de las células de la testa, escultura de la pared periclinal y la RHM de las semillas de Neobuxbaumia y Carnegiea. Ver cuadro 2 para las abreviaturas.

\begin{tabular}{|c|c|c|c|c|c|c|}
\hline Especies & $\begin{array}{c}\text { Límites } \\
\text { anticlinales }\end{array}$ & $\begin{array}{l}\text { Escultura de la } \\
\text { pared anticlinal }\end{array}$ & Micro-relieve & Intersticios & $\begin{array}{c}\text { Posición relativa } \\
\text { a la } \mathrm{RHM}\end{array}$ & $\begin{array}{c}\text { Forma del } \\
\text { complejo RHM }\end{array}$ \\
\hline N. euphorbioides & $\mathrm{AC}$ & $\mathrm{P}$ & $\mathrm{L}$ & A & SU & $\mathrm{O}$ \\
\hline N. laui & I & DA & $\mathrm{L}$ & A & SU & $\mathrm{O}$ \\
\hline N. macrocephala & $\mathrm{AC}$ & $\mathrm{P}$ & $\mathrm{L}$ & A & SU & $\mathrm{O}$ \\
\hline N. mezcalaensis & I & DB & $\mathrm{L}$ & $A$ & SU & $\mathrm{O}$ \\
\hline N. multiareolata & I & DB & $\mathrm{L}$ & $\mathrm{P}$ & SU & $\mathrm{O}$ \\
\hline N. polylopha & $\mathrm{AC}$ & $\mathrm{P}$ & $\mathrm{EF}$ & A & SU & $\mathrm{O}$ \\
\hline N. scoparia & LL & $\mathrm{P}$ & $\mathrm{L}$ & A & SU & $\mathrm{O}$ \\
\hline N. squamulosa & $\mathrm{AC}$ & DB & $\mathrm{EF}$ & A & SU & $\mathrm{O}$ \\
\hline N. tetetzo & LL & $\mathrm{P}$ & $\mathrm{L}$ & A & SU & $\mathrm{O}$ \\
\hline C. gigantea & LL & $\mathrm{P}$ & $\mathrm{EF}$ & $A$ & FIM & CE \\
\hline
\end{tabular}
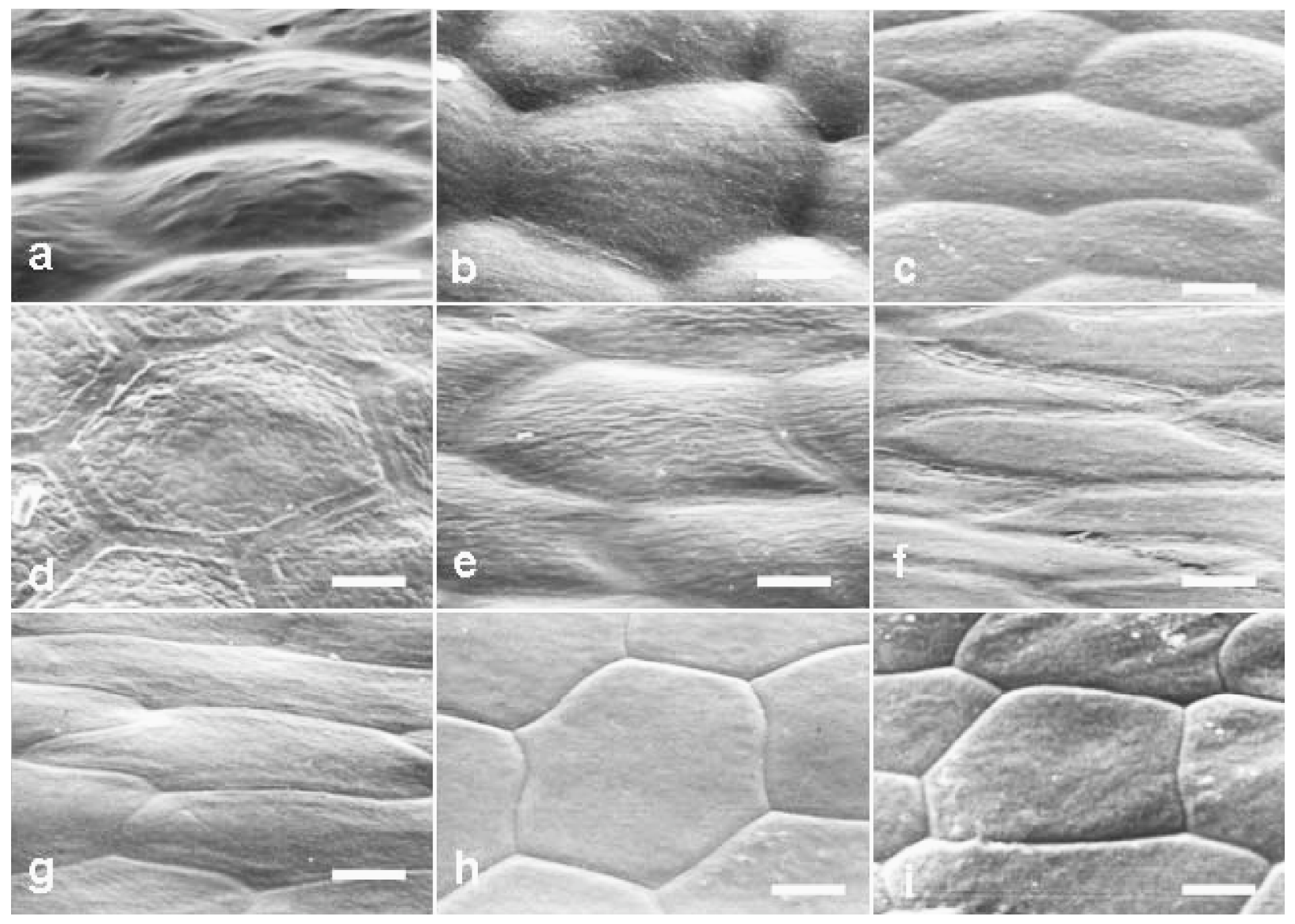

Figura 3. Microfotografías MEB de la forma celular y microrelieve en la región lateral de las células. a. Neobuxbaumia laui, Jardín Etnobotánico de Oaxaca 1417. b. N. multiareolata, Arroyo-Cosultchi 8 (CHAPA). c. N. mezcalaensis, Flores s.n. (IZTA). d. N. poly lopha, Arroyo-Cosultchi 22 (CHAPA). e. N. squamulosa, Flores s.n. (IZTA). f. N. euphorbioides, Flores s.n. (IZTA). g. N. macrocephala, Flores s.n. (IZTA). h. N. tetetzo, S. Arias 831 (MEXU). i. $N$. scopari a, Bravo-Hollis 71 (MEXU). Escalas: b = 20 m, a, c-e, h= $50 \mathrm{~m}, \mathrm{i}, \mathrm{f}=100 \mathrm{~m}$. 

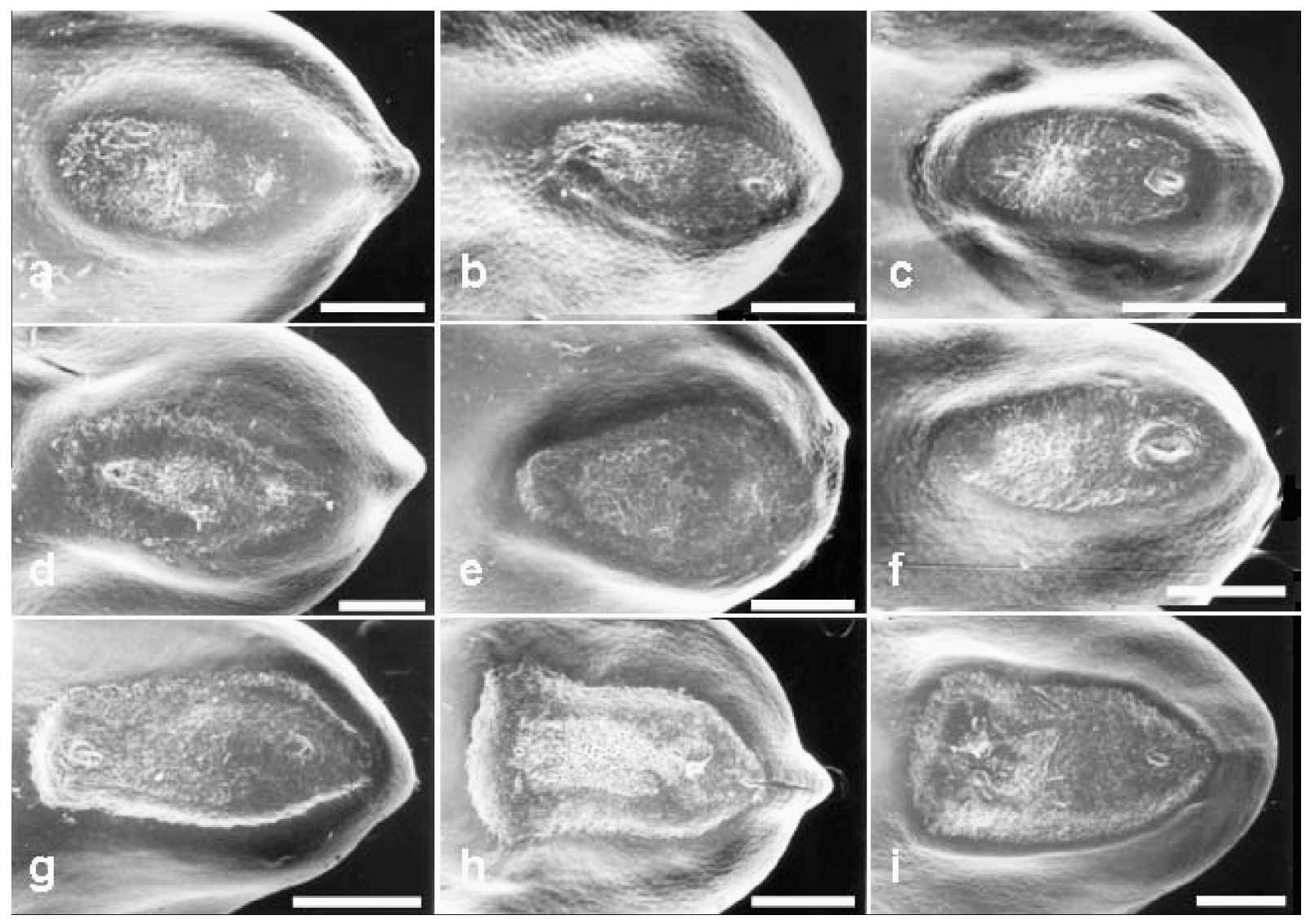

Figura 4. Microfotografías MEB de la region hilo-micropilar de la semilla. a. Neobuxbaumia macrocephala, Flores s.n. (IZTA). b. N. scoparia, Bravo-Hollis 71 (MEXU). c. N. euphorbioides, Flores s.n. (IZTA). d. N. polylopha, Flores s.n. (IZTA). e. N. squamulosa, Flores s.n. (IZTA). f. N. tetetzo, S. Arias 831 (MEXU). g. N. multiareolata, Arroyo-Cosultchi 8 (CHAPA). h. N. mezcalaensis, Flores s.n. (IZTA). i. N. laui, Jardín Etnobotánico de Oaxaca 1417. Escalas: a,b = 300 m, c, e, g, h, i = $500 \mathrm{~m}, \mathrm{~d}, \mathrm{f}=250 \mathrm{~m}$.

do en $N$. scoparia, $N$. tetetzo y $C$. gigantea, y acanalado en N. euphorbioides, N. macrocephala, N. polylopha y $N$. squamulosa (figuras 2, 3). Además, el margen de las células es recto y con intersticios diferenciados exclusivamente en $N$. multiareolata (figura 3b).

Estructura de la pared periclinal._La pared periclinal es lisa, excepto en $N$. polylopha, $N$. squamulosa y C. gigan tea, con micro-estriaciones finas (figuras $2 \mathrm{~b}, 3 \mathrm{~d}$, e); sin convexidad excepto en $N$. mezcalaensis, $N$. multiareolata y $N$. squamulosa, en las que se presenta convexidad de domo bajo, y $N$. laui de domo alto (cuadro 4; figura 3).

Región hilo-micropilar (RHM).-La orientación de la RHM es oblicua; la posición es superficial en todas las especies de Neobuxbaumia e impresa en $C$. gigantea (figuras 2,4). La configuración del hilo-micrópilo tiene un tejido esclerificado. Además, la RHM es de forma ovalada en todas las especies de Neobuxbaumia (figura 4) y de forma de cerradura en $C$. gigantea (figura 2c).

Análisis de similitud. El fenograma separa dos grupos a un nivel de corte de 0.28 (figura 5). El primer grupo (A) inclu- ye a las nueves especies de Neobuxbaumia agrupadas por presentar semillas ovoides y una posición relativa a la RHM superficial. Este grupo se subdivide en dos por el tamaño de las semillas. El primer subgrupo (a1) consiste de tres especies, $N$. euphorbioides, $N$. macrocephala y N. sco paria, que son las que presentan las menores dimensiones (largo $<2.5 \mathrm{~mm}$, ancho $<2.0 \mathrm{~mm}$, área $<4.0 \mathrm{~mm}^{2}$ ); el segundo subgrupo (a2) contiene el resto de las especies de Neobuxbaumia con las semillas más grandes (largo $>2.50$ $\mathrm{mm}$, ancho $>2.00 \mathrm{~mm}$, área $>4.0 \mathrm{~mm}^{2}$ ). En el subgrupo (a2), el par de especies con mayor similitud son N. mezca laensis y $N$. multiareolata. El segundo grupo (B) está compuesto por Carnegiea gigantea y Pachycereus weberi. Estas dos especies poseen semillas ovadas y una posición relativa a la RHM finamente impresa.

\section{Discusión}

En este trabajo se describen y comparan por primera vez las semillas de todas las especies reconocidas de Neobuxbaumia. Las nueves especies tienen semillas largas a muy 


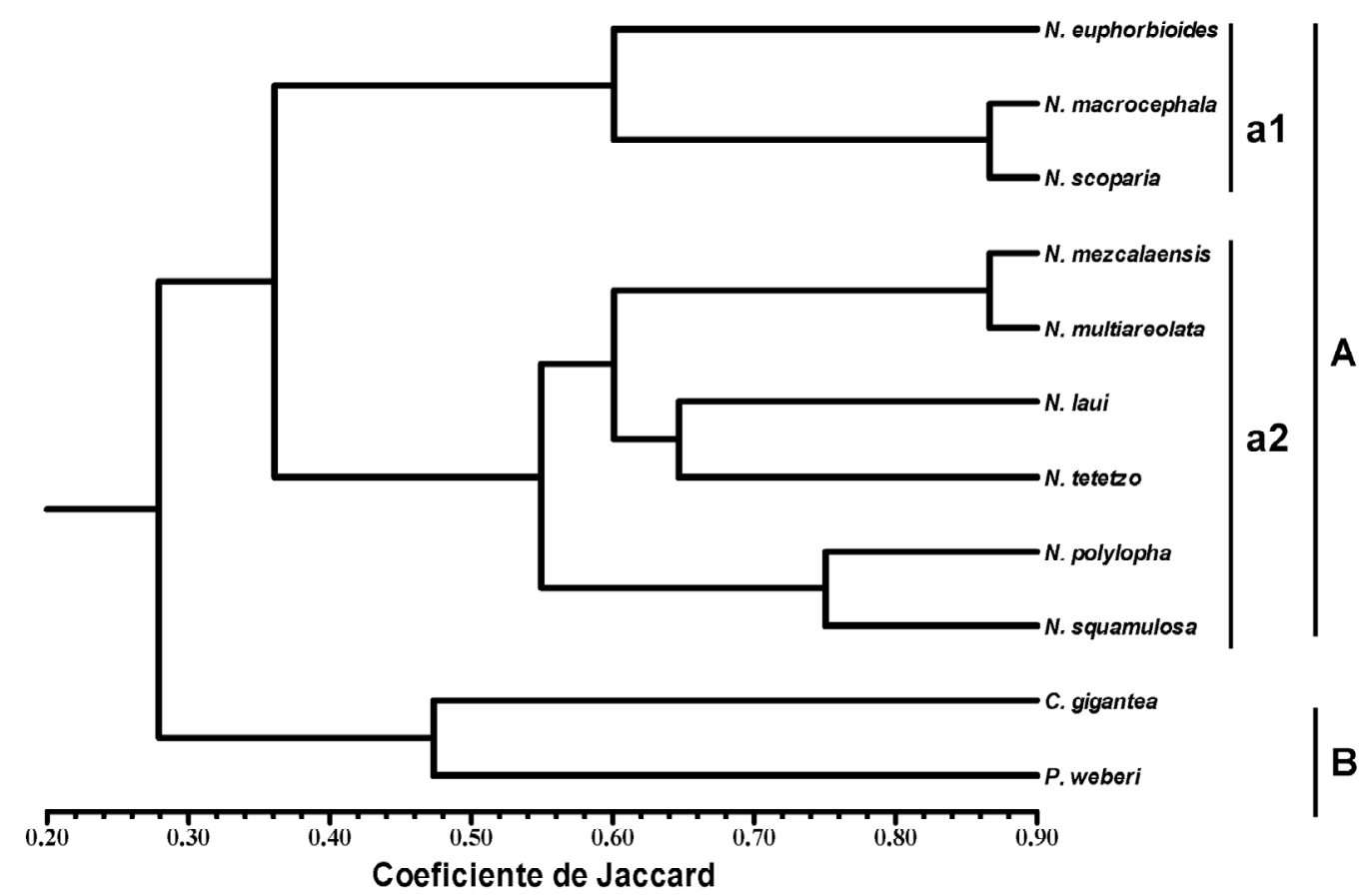

Figura 5. Fenograma de las especies de Neobuxbaumia con base en 14 caracteres de la semilla. Las letras mayúsculas indican grandes grupos y las letras minúsculas los dos subgrupos del grupo A.

largas, asimétricas, lustrosas, lisas y con una quilla en la periferia, con el borde ligeramente expandido alrededor del hilo, la RHM oblicua, superficial y el complejo de forma ovalada. Lo lustroso de las semillas, su superficie lisa y la presencia de quilla en las especies de Neobuxbaumia son tres rasgos que comparten con las semillas de los otros miembros de la subtribu Pachycereinae, incluyendo a Carnegiea gigantea (Gibson y Horak, 1978; Terrazas y Loza-Cornejo, 2002; Arias y Terrazas, 2004, 2006). Sin embargo, la presencia de la quilla no es un carácter exclusivo de la subtribu Pachycereinae, porque las semillas de Stenocereus (subtribu Stenocereinae; Arroyo-Cosultchi et al., 2006) y de otros géneros de las tribus Cereeae y Trichocereeae (Barthlott y Hunt, 2000) también la presentan. Al igual que en Stenocereus, la posición donde termina la quilla es variable: en Neobuxbaumia predomina su terminación en la región ventral, posición registrada sólo para S. zopilotensis (Arroyo-Cosultchi et al., 2006).

El fenograma separa las especies de Neobuxbaumia en dos grupos con base principalmente en su tamaño. Las tres especies con tamaños menores que $2.5 \mathrm{~mm}$ de largo y 2.0 $\mathrm{mm}$ de ancho ( $N$. euphorbioides, $N$. macrocephala y $N$. scoparia) también tienen la escultura de la pared periclinal plana y el microrelieve liso. Sin embargo, $N$. euphorbioides se distingue de las otras dos por tener semillas de color café, con células de forma poligonal-alargada y los límites de sus paredes anticlinales acanalados.

En el segundo grupo, las seis especies de Neobuxbaumia se agrupan por su tamaño, el cual fluctúa entre 2.68 1.85 mm en $N$. polylopha y $4.00 \quad 3.00$ en $N$. laui, además de tener células de forma poligonal-isodiamétrica. Aunque el tamaño y la forma celular se comparten con Backebergia militaris (Audot) Bravo ex Sánchez-Mej., Cephalocereus columna-trajani (Karw. ex Pfeiff.) K.Schum., Pterocereus gaumeri (Britton et Rose) T.MacDoug. et Miranda y las especies de Lemaireocereus, Marshallocereus y Pachycereus (Arias y Terrazas, 2004; Bárcenas-Argüello, 2006; actualización de nombres según Arias y Terrazas, sometido), difieren en la combinación de los atributos: límites de sus paredes anticlinales, escultura de la pared periclinal y microrelieve. Por ejemplo, $N$. mezcalaensis y $N$. multiareolata tienen los límites de las paredes anticlinales invisibles y la pared periclinal con domo bajo, pero la presencia de intersticios es exclusiva de $N$. multiareolata.

Neobuxbaumia polylopha y N. squamulosa presentan en común semillas negras, con límites anticlinales acanalados y microestriaciones finas, pero difieren entre sí en la posición de la quilla y la escultura la pared de la célula. En el caso de $N$. laui y N. tetetzo, este par de especies presentan las semillas más disímiles del grupo a2, ya que difieren en el color y los límites periclinales de las células de la región lateral; además, $N$. laui se distingue por ser la única especie del género con la pared periclinal de las células con convexidad de domo alto.

Arias y Terrazas (2004) señalan que se observó la ausencia del borde de la testa en la RHM en Pseudomitrocereus fulviceps (F.A.C.Weber ex K.Schum.) Bravo et Buxb., pudiendo interpretarse como un estado compartido con las 
especies de Cephalocereus y Neobuxbaumia. Sin embargo, este supuesto no se cumple para las especies de Neobuxbaumia, donde se reconoce que el borde es ligeramente expandido. Por ello, este estado de carácter se comparte con las especies de Marshallocereus y Pachycereus. El borde ampliamente expandido aquí descrito para Carnegiea gigantea se comparte con Lophocereus gatesii M.E.Jones y L. schottii (Engelm.) Britton et Rose (Arias y Terrazas, 2004).

En conclusión, los resultados permiten diferenciar a las especies de Neobuxbaumia de Carnegiea gigantea, por la combinación de semillas de forma ovoide con borde de la testa ligeramente expandido alrededor del hilo, la posición relativa a la RHM superficial y el complejo en forma de cerradura. Sin embargo, no se encontró ningún carácter que sirva como sinapomorfia potencial para el género Neobuxbaumia, ya que prácticamente todos los caracteres aquí analizados de la semilla se comparten con miembros de otros géneros de la subtribu Pachycereinae.

\section{Agradecimientos}

Se agradece el apoyo de CONACYT a través de una beca para realizar estudios de maestría al primer autor (No. 190345). Los autores agradecen a Cesario Catalán, Leobardo Sánchez y Loraine Matias su ayuda en el trabajo de campo, al curador de MEXU el permiso para remover semillas de algunos especímenes; a César Flores, Ligia Esparza y a las autoridades del Jardín Etnobotánico de Oaxaca por proporcionarnos muestras de semillas; a Berenit Mendoza del Instituto de Biología (Universidad Nacional Autónoma de México) por la asistencia técnica en el microscopio electrónico de barrido y a Julio César Montero del Instituto de Biología (UNAM), por digitalizar las imágenes; al Dr. Ken Cameron y un revisor anónimo por sus sugerencias y comentarios favorables.

\section{Literatura citada}

Arias S. y Terrazas T. 2004. Seed morphology and variation in the genus Pachycereus (Cactaceae). Journal of Plant Research 117:277-289.

Arias S. y Terrazas T. 2006. Análisis cladístico del género Pachycereus (Cactaceae) con caracteres morfológicos. Brittonia 58:197-216.

Arias S. y Terrazas T. Sometido. Taxonomic revision of Pachycereus (Cactaceae).

Arias S., Terrazas T., Arreola-Nava H.J., Vázquez-Sánchez M. y Cameron K.M. 2005. Phylogenetic relationships in Peniocereus (Cactaceae) inferred from plastid DNA sequence data. Journal of Plant Research 118:317-328.

Recibido: $1^{\circ}$ de junio de 2007

Aceptado: 29 de octubre de 2007
Arias S., Terrazas T. y Cameron K.M. 2003. Phy l ogenetic analysis of Pachycereus (Cactaceae, Pachycereeae) based on chloroplast and nu clear DNA sequences. Systematic Botany 28:547-557.

Arroyo-Cosultchi G., Terrazas T., Arias S. y Arreola-Nava H.J. 2006. The systematic significance of seed morphology in Stenocereus (Cactaceae). Taxon 55:983-992.

Bárcenas-Arguiello M.L. 2006. Filogenia del género Cephalocereus Pfeiff. (Cactaceae) sensu Anderson con base en caracteres estructurales. Tesis de Maestría, Colegio de Postgraduados, Montecillo, Estado de México, 116 pp.

Barthlott W. y Hunt D.R. 2000. Seed diversity in the Cactaceae subfamily Cactoideae. Succulent Plant Research 5:1-173.

Barthlott W. y Voit G. 1979. Mikromorphologie der Samenschalen und Taxonomie der Cactaceae: Ein raster-elektronenmikroskopischer Überblick. Plant Systematics and Evolution 132:205-229.

Bravo-Hollis H. 1978. Las Cactáceas de México. Vol. 1. Universidad Nacional Autónoma de México, México, D.F.

Crisci J.V. y López-Armen gol M.F. 1983. Introducción a la Teoría y Práctica de la Taxonomía Numérica. Secretaría General de la Organización de los Estados Americanos, Washington, D.C.

Gibson A.C. y Horak K.E. 1978. Systematic anatomy and phylogeny of Mexican columnar cacti. Annals of the Missouri Botanical Garden 65:999-1057.

Guzmán U., Arias S. y Dávila P.D. 2003. Catálogo de Cactáceas Mexicanas. Universidad Nacional Autónoma de México y Comisión Nacional para el Conocimiento y Uso de la Biodiversidad, México, D.F.

Heath P.V. 1992. The restoration of Rathbunia. Calyx 2:102-104.

Hunt D.R. 2006. The New Cactus Lexicon. International Cactaceae Systematics Group, Milborne Port, Reino Unido.

Hunt D.R. y Taylor N.P. 1990. The genera of Cactaceae: progress towards consensus. Bradleya 8:85-107.

Lau A.B. 1994. Neobuxbaumia sanchezmejoradae Lau. Cactáceas y Suculentas Mexicanas 39:3-7.

Media Cybernetics. 1997. Image-Pro Plus Reference Guide. Version 3.01 for Windows, Media Cybernetics, Silver Spring, Maryland.

Rohlf F.J. 1997. NTSYSpc. Numerical Taxonomy and Multivariate Analysis System. Version 2.0. Applied Biostatistics Inc., Nueva York.

SAS Institute. 1989. SAS/STAT User's Guide, Release 6.03 Edition. SAS Institute Inc., Cary.

Scheinvar L. y Sánchez-Mejorada H. 1990. Neobuxbaumia squa mulosa, sp. nov. Cactáceas y Suculentas Mexicanas 35:13-18.

Sneath P.H. y Sokal R.R. 1973. Numerical Taxonomy. The Principles and Practices of Numerical Classification. W.H. Freeman and Company, San Francisco.

Te rrazas T. y Loza-Cornejo S. 2002. Phy logenetic relationships of Pachycereeae: a cladistic analysis based on anatomicalmorphological data. En: Fleming T.H. y Valiente-Banuet A. Eds. Evolution, Ecology, and Conservation of the Columnar Cacti and their Mutualisms, pp 66-86, Arizona University Press, Tucson. 
Apéndice 1. Especies y caracteres usados para generar la matriz de similitud. El nombre de los caracteres se proporciona en el cuadro 2. $N E U=$ Neobuxbaumia euphorbioides; $N L A=N$. laui; $N M A=N$. macrocephala; $N M E=N$. mezcalaensis; $N M U=$ N. multiareolata, $N P O=N$. polylopha; $N S C=N$. scoparia; $N S Q=N$. squamulosa; NTE = N. tetetzo; CGI = Carnegiea gigantea; $P W E=$ Pachycereus weberi.

\begin{tabular}{|c|c|c|c|c|c|c|c|c|c|c|c|}
\hline & $N E U$ & $N L A$ & $N M A$ & NME & NMU & $N P O$ & NSC & $N S Q$ & NTE & CGI & $P W E$ \\
\hline 1 & 0 & 0 & 0 & 0 & 0 & 0 & 0 & 0 & 0 & 1 & 1 \\
\hline 2 & 1 & 1 & 1 & 1 & 1 & 1 & 1 & 1 & 1 & 0 & 0 \\
\hline 3 & 0 & 1 & 1 & 0 & 0 & 1 & 1 & 1 & 0 & 1 & 1 \\
\hline 4 & 1 & 0 & 0 & 1 & 1 & 0 & 0 & 0 & 1 & 0 & 0 \\
\hline 5 & 1 & 1 & 0 & 0 & 0 & 1 & 0 & 0 & 1 & 0 & 0 \\
\hline 6 & 0 & 0 & 1 & 1 & 1 & 0 & 1 & 1 & 0 & 1 & 1 \\
\hline 7 & 1 & 1 & 1 & 1 & 1 & 1 & 1 & 1 & 1 & 0 & 1 \\
\hline 8 & 0 & 0 & 0 & 0 & 0 & 0 & 0 & 0 & 0 & 1 & 0 \\
\hline 9 & 0 & 1 & 0 & 1 & 1 & 1 & 1 & 1 & 1 & 0 & 0 \\
\hline 10 & 1 & 0 & 1 & 0 & 0 & 0 & 0 & 0 & 0 & 1 & 1 \\
\hline 11 & 0 & 1 & 0 & 1 & 1 & 0 & 0 & 0 & 0 & 0 & 0 \\
\hline 12 & 1 & 0 & 0 & 0 & 0 & 1 & 0 & 1 & 0 & 0 & 0 \\
\hline 13 & 0 & 0 & 1 & 0 & 0 & 0 & 1 & 0 & 1 & 1 & 1 \\
\hline 14 & 1 & 0 & 1 & 0 & 0 & 1 & 1 & 0 & 1 & 1 & 1 \\
\hline 15 & 0 & 0 & 0 & 1 & 1 & 0 & 0 & 1 & 0 & 0 & 0 \\
\hline 16 & 0 & 1 & 0 & 0 & 0 & 0 & 0 & 0 & 0 & 0 & 0 \\
\hline 17 & 1 & 1 & 1 & 1 & 1 & 1 & 1 & 1 & 1 & 0 & 0 \\
\hline 18 & 0 & 0 & 0 & 0 & 0 & 0 & 0 & 0 & 0 & 1 & 1 \\
\hline 19 & 1 & 1 & 1 & 1 & 1 & 1 & 1 & 1 & 1 & 0 & 1 \\
\hline 20 & 0 & 0 & 0 & 0 & 0 & 0 & 0 & 0 & 0 & 1 & 0 \\
\hline 21 & 0 & 1 & 0 & 1 & 1 & 1 & 0 & 1 & 1 & 0 & 1 \\
\hline 22 & 1 & 0 & 1 & 0 & 0 & 0 & 1 & 0 & 0 & 1 & 0 \\
\hline 23 & 0 & 1 & 0 & 1 & 1 & 1 & 0 & 1 & 1 & 0 & 1 \\
\hline 24 & 1 & 0 & 1 & 0 & 0 & 0 & 1 & 0 & 0 & 1 & 0 \\
\hline 25 & 0 & 1 & 0 & 1 & 1 & 1 & 0 & 1 & 1 & 0 & 1 \\
\hline 26 & 1 & 0 & 1 & 0 & 0 & 0 & 1 & 0 & 0 & 1 & 0 \\
\hline 27 & 1 & 1 & 1 & 1 & 0 & 1 & 1 & 1 & 1 & 1 & 1 \\
\hline 28 & 0 & 0 & 0 & 0 & 1 & 0 & 0 & 0 & 0 & 0 & 0 \\
\hline 29 & 1 & 1 & 1 & 1 & 1 & 0 & 1 & 0 & 1 & 0 & 0 \\
\hline 30 & 0 & 0 & 0 & 0 & 0 & 1 & 0 & 1 & 0 & 1 & 1 \\
\hline
\end{tabular}

